Proceedings of Eurohaptics, LNCS 5024, Springer-Verlag, pp. 361-366.

http://www.disam.upm.es/ eurohaptics2008/

\title{
Haptic Perception of Real and Virtual Curvature
}

\author{
Maarten W.A. Wijntjes ${ }^{1}$ and Akihiro Sato ${ }^{2}$ \\ Astrid M.L. Kappers ${ }^{1}$, and Vincent Hayward ${ }^{2}$ \\ ${ }^{1}$ Helmholtz Institute, Utrecht University, the Netherlands \\ ${ }^{2}$ Haptics Laboratory, Centre of Intelligent Machines, McGill University, Canada
}

\begin{abstract}
In this study we compared human discrimination performance for real and virtual curved shapes. To simulate a curved shape we used a device that could independently orient and elevate a moving surface that was in contact with an exploring finger. Thus, the geometry was preserved up to the first order in the virtual shape. In our experiment we found that this preservation was indeed sufficient: discrimination thresholds were similar for the real and virtual conditions. Our results were also in line with previous curvature studies performed with real stimuli.
\end{abstract}

\section{Introduction}

In the present study, we compared haptic curvature discrimination performance between virtual and real stimuli. We used a type of device nicknamed a 'Morpheotron' [1]. Several versions were built for different purposes. The version we used here was optimized specifically for the present study. The workspace of the original device was increased from $25 \mathrm{~mm}$ to $160 \mathrm{~mm}$. This version can simulate the height of the place of contact in addition to the orientation of the surface of contact (slope). We compared discrimination performance on the new Morpheotron with real stimuli previously used by Pont et al. [2].

\section{Methods}

The stimuli were cylindrically curved strips of radius $R$ that all had the same height at the apex. The height, $z$, and the slope, $\theta$, as a function of horizontal position, $x$, are described by $z=\left(\sqrt{1-(C x)^{2}}-1\right) / C$ and $\theta=\sin ^{-1}(C x)$ where the curvature $C=1 / R$. The function $z(x)$ describes a path of constant curvature with $z(0)=0$ for all $C$. The servo-mechanism described in one of the following sections is controlled according to the formulas for $z(x)$ and $\theta(x)$.

Real stimuli We used the same curved PVC blocks that were used by Pont et al. [2]. They were $20 \mathrm{~cm}$ long and $2 \mathrm{~cm}$ wide. All stimuli were $5 \mathrm{~cm}$ high at their highest (convex) or lowest (concave) point. Scanning was limited at each end by small curved barriers to avoid unwanted cueing. The stimuli were disposed such that lateral movements on the real stimuli were similar to those generated by the device. 
Proceedings of Eurohaptics, LNCS 5024, Springer-Verlag, pp. 361-366.

http://www.disam.upm.es/ eurohaptics2008/

Apparatus The apparatus orients and elevates a moving surface in contact with the finger of a subject during lateral exploration. Such an ability makes it possible to simulate a virtual object [1]. The present variant makes it possible to control the elevation, $z$, in addition to the orientation, $\theta$ of the region where the virtual object is touched. For experimental simplicity, it has only one orientation angle rather than two in the original device, restricting exploration to one curvilinear dimension. Such an apparatus makes it possible to separate information made available to an observer in terms of cues related to the height of the object (zeroth order information) from cues related to the slope of the object where it is touched (first order information) [2]. This can be accomplished with a flat plate that can be freely moved sideways by the observer. During exploration the plate can be independently oriented and elevated under servo control. Please refer to Fig. 1 for the labeling of the degrees-of-freedom (DOFs).

Construction Referring to Fig. 1, the apparatus was a three DoF robotic-type mechanism. It included a powerful motor A (brush type DC motor model RDM103, Servo Systems Co., Montville, NJ, USA) that vertically drove a platform B via an anti-backlash lead-screw C (Model $12.7 \mathrm{~mm} / \mathrm{rev}$., MLPS-2-10, Servo Systems Co., Montville, NJ, USA). A low-friction linear guide D (Model SBG10-3, LM76 Linear Motion Bearings, E. Longmeadow, MA, USA) was mounted on the platform allowing a carriage to move freely. Its position was measured by an encoder E. The custom-made orienting mechanism mounted on the carriage included a capstan torque amplification (1:20) mechanism $\mathrm{F}$ driven by a miniature DC motor G (Maxon A-max $19 \mathrm{~mm} 2.5 \mathrm{~W}$, Maxon Motors AG, Sachseln, Switzerland). Both electric motors were fitted with 400 CPR shaft encoders and driven by power amplifiers. Control was performed at a fixed update rate of $5 \mathrm{kHz}$ on a PC running Linux kernel 2.6 and the Xenomai real-time framework. Input/Output was performed by an AD/DA and counter ISA board (Model II, Servo to Go, Inc., Indianapolis, IN, USA).
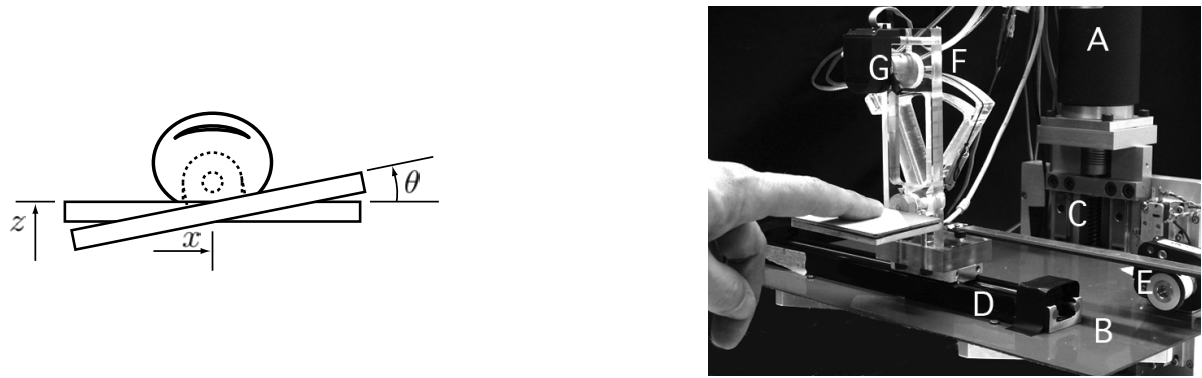

Fig. 1. Left: Degrees of freedom. Elevation $z$ and orientation $\theta$ are servo-controlled. A cross-section of a fingertip rests on the plate. The dashed circle indicates center of rotation. Right: View of the apparatus. An index finger interacts with a plate without slip. When a subject explores a virtual surface laterally, the height and the orientation of the contact surface can be independently specified. See text. 
Proceedings of Eurohaptics, LNCS 5024, Springer-Verlag, pp. 361-366.

http://www.disam.upm.es/ eurohaptics2008/

Control The control system is illustrated in Fig. 2. It takes as input the horizontal plate position, $x$, and performs kinematic transformations to reproduce the curvature, $C$, of the cylindrical virtual object to be simulated. The outputs, $z$ and $\theta$, are supplied as setpoints to two local servo loops. Friction in the antibacklash leadscrew results in significant steady state error, even if the loop gain is high. The standard control design to deal with this problem is to include an integral gain in the loop. However, a well tuned integral gain has a long time constant. A slow-acting integral gain would have a detrimental effect on our experiment. During slow movements, the integral gain would cause the plate to creep slowly toward its setpoint, resulting in an unnatural tactile experience.

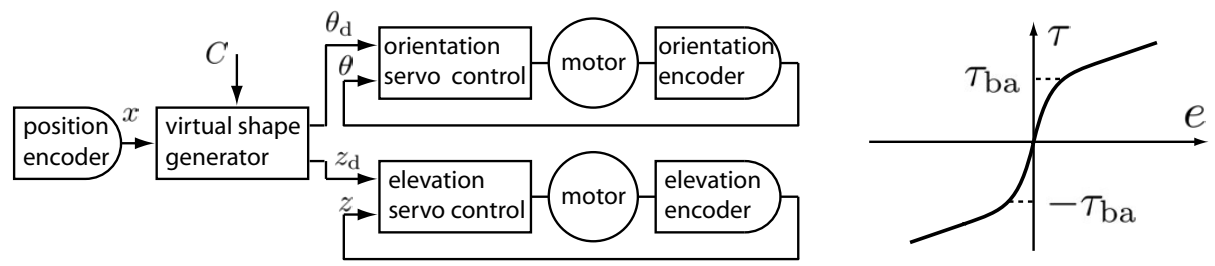

Fig. 2. Left: Degrees of freedom. Elevation $z$ and orientation $\theta$ are servo-controlled, $z_{d}$ and $\theta_{d}$ are the desired values. Right: Shape of the nonlinear gain $K_{\mathrm{p}}(e)$.

To increase accuracy without an integral term, model-based friction compensation could be an option, but that would be difficult to implement. In addition, it is doubtful that our sensors would achieve the required resolution [3]. Since the prospects for increasing performance with linear feedback are limited, we turned our attention to a class of nonlinear controllers - nonlinear PD controllers [4] which are known to be able to deal with dry friction while preserving a smooth response. These controllers come in many types. Here, we implemented a very simple version which can be expressed as a nonlinear proportional error gain of the form $K_{\mathrm{p}}(e)=a\left(\frac{2}{1+\exp (b e)}-1\right)+c e$, where $a=\tau_{\text {ba }}, b=\frac{2}{\tau_{\mathrm{ba}}}\left(K_{\text {low }}-K_{\mathrm{high}}\right)$, $c=K_{\text {low }}$. This expression introduces two gains with a smooth transition in between, see Fig. 2, to prevent the onset of limit cycles.

The intuition behind this choice comes from two observations. Firstly, when the error is small, control stiffness is increased, which is a usual method to compensate for friction. The second intuition is that the assembly of two gains in a single curve resembles a saturation plus a gain. This curve is, in essence, an inverse model for a transmission with friction [5]. In fact, points $\pm \tau_{\text {ba }}$ in Fig. 2 have a nice physical interpretation: they are the breakaway torques, that is, the torques needed to cause the transmission to transition from the stuck state to the sliding state, which will be achieved even though the error is small. With the addition of a derivative term to damp the response and an additional torque to compensate for gravity $\left(\tau_{g}\right)$, the nonlinear servo controller is given by

$$
e_{z}=z_{\mathrm{d}}-z, \tau_{z}=K_{\mathrm{p} z}\left(e_{z}\right)+K_{\mathrm{d} z} \dot{e}_{z}+\tau_{g}, e_{\theta}=\theta_{\mathrm{d}}-\theta, \tau_{\theta}=K_{\mathrm{p} \theta} e_{\theta}+K_{\mathrm{d} \theta} \dot{e}_{\theta}
$$


Proceedings of Eurohaptics, LNCS 5024, Springer-Verlag, pp. 361-366.

http://www.disam.upm.es/ eurohaptics2008/

Performance We measured the total tracking error between actual and the desired elevation and orientation with the device tracking a reference curvature of $2 \mathrm{~m}^{-1}$ for different exploration speeds $(40,80$, and $160 \mathrm{~mm} / \mathrm{s}$ ). Fig. 3 (left panel) shows a trajectory plot recorded from a movement with moderate velocity $(80 \mathrm{~mm} / \mathrm{s})$. Both the elevation and the orientation were smoothly tracking the desired values. There was a small delay, $d \approx 23 \mathrm{~ms}$ resulting from the dynamic tracking error. Since this error was too small to be graphically appreciated, Fig. 3 (right panel) shows the error of the shape actually displayed and computed once the delay was corrected for. The robustness of the control algorithms can be appreciated from the fact that the error is by-and-large independent from speed, despite a systematic bias. Nevertheless, the static shape error never exceeded 100 microns in height and $0.2^{\circ}$ in orientation under any operating condition.
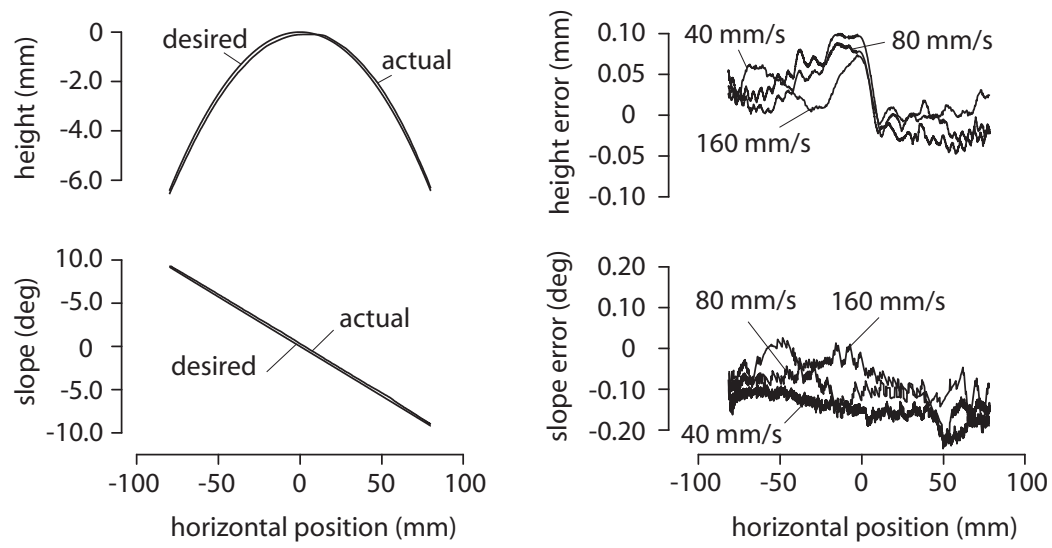

Fig. 3. Left: Desired and actual elevation and orientation for $C=2 \mathrm{~m}^{-1}$. The height is zero in the middle and the orientation changes from a positive to a negative value. Right: Shape errors for height and slope after delay correction and for three different exploration speeds.

Participants Eight volunteers participated in the experiment. They were recruited from the McGill University student population and were compensated for their time. According to the Coren handedness test [6], seven were strongly right handed, and one was weakly right handed.

Experimental procedure and design We used a 2-AFC design with the method of constant stimuli. The observers were presented with 2 stimuli, one reference and one test. They had to judge which of the two felt more convex. An illustration of what a convex shape looks like was posted as a reminder. The reference stimulus was always flat, i.e. $C=0 \mathrm{~m}^{-1}$. Subjects were seated behind a curtain that hid the apparatus, the stimuli and their forearm. They wore isolating headphones throughout the experiment. 
Proceedings of Eurohaptics, LNCS 5024, Springer-Verlag, pp. 361-366.

http://www.disam.upm.es/ eurohaptics2008/

We used a blocked design in which the order of the conditions (real and virtual) was counterbalanced. Within blocks, the order of test and reference stimulus was randomised. We started each block with two repetitions of $c_{t}=$ $\{-2,-1.8, \ldots,-0.2,0.2, \ldots, 1.8,2\}$ presented in random order. From the results, we analysed the responses and designed an additional, larger, final set of 12 different test curvatures and 8 repetitions. It gave us the opportunity to adjust the stimulus set. For the analysis we used the responses of test curvatures which were both in the small and large set. A total of 120 responses were used for each threshold. The data were then fitted to a cumulative Gaussian $f(c, \sigma)=$ $0.5 \operatorname{erf}(c / \sqrt{s} \sigma)+0.5$ in which $c$ is the test curvature, $\mu=0$ is the reference curvature and $\sigma$ is the $84 \%$ discrimination threshold. We used the maximum likelihood estimation procedure to fit the functions. This procedure takes into account the binomial distribution of the responses.

\section{Results}

Individual thresholds are presented in Fig. 4 . The average thresholds were $0.44 \mathrm{~m}^{-1}$ and $0.45 \mathrm{~m}^{-1}$ for the virtual and real conditions, respectively. A paired $t$-test $(t(7)=-0.255, p=0.806)$ showed that this difference was not significant. Since both convex and concave stimuli were used it was possible to analyze the data for each case and investigate whether the thresholds were similar. We used paired $t$-tests to compare the convex and concave discrimination thresholds. For neither of the conditions (real or virtual) we found a significant effect. This reflects that humans may be equally sensitive to convex and concave shapes, as was previously found for passive cutaneous curvature discrimination [7].

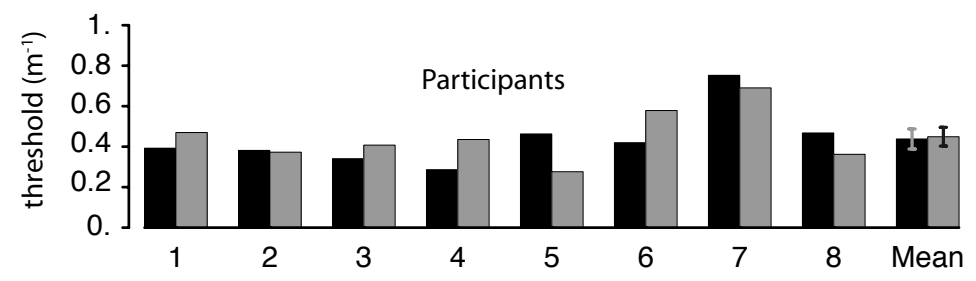

Fig. 4. Individual thresholds for all participants. Black bars denote the virtual condition, the gray bars the real stimuli. Mean results and standard errors are shown.

\section{Discussion}

Although it is impossible to confirm a null-hypothesis, our results indicate that human curvature sensitivity is essentially similar for virtual and real shapes. If there is any difference between the two stimuli, this difference is very small. The average thresholds that we found were close to $0.5 \mathrm{~m}^{-1}$. These results are similar to those found by Pont et al. [2], using a lateral motion range of $20 \mathrm{~cm}$. Our result is statistically stronger than a mere comparison of thresholds between different 
Proceedings of Eurohaptics, LNCS 5024, Springer-Verlag, pp. 361-366.

http://www.disam.upm.es/ eurohaptics2008/

studies. We can thus conclude that Morpheotron-type devices simulate curvature within the range of human haptic discrimination thresholds, i.e., virtual shapes are perceptually similar to real shapes. Our finding is in line with Provancher et al. [8] who investigated curvature discrimination with four nonzero reference curves (between 25 and $100 \mathrm{~m}^{-1}$ ) for real and virtual stimuli.

When using haptic devices, one should be aware that unwanted cues such as vibrations, control instabilities, stick-slip, mechanical artifacts, or even auditory signals can be informative to the participants. For example, if an observer can detect a transient vibration and is asked which of two stimuli is flat, he may use this vibration as a cue instead of the perceived slope and height. To overcome this problem we used a $2 \mathrm{AFC}$ design and asked the participants which stimulus was more convex. They did not have to detect which stimulus was non-zero but had to detect the sign of the curvature. This procedure eliminated any possibility that unwanted cues could influence the discrimination threshold. It was therefore not necessary to test whether these cues were in fact present in the experiment. Furthermore, the device performance was well within the limits of human detectability. The mean threshold expressed in slope differences is $4.5^{\circ}$, and in base-to-apex height $2 \mathrm{~mm}$; both are much larger than machine precision.

Acknowledgements This research was supported by a grant from the Netherlands Organisation for Scientific Research (NWO), a grant from the IEEE TC on Haptics Student Exchange Program, and a Discovery Grant from the Natural Sciences and Engineering Council of Canada (NSERC).

\section{References}

1. Dostmohamed, H., Hayward, V.: Trajectory of contact region on the fingerpad gives the illusion of haptic shape. Experimental Brain Research 164(3) (2005) 387-394

2. Pont, S.C., Kappers, A.M.L., Koenderink, J.J.: Similar mechanisms underlie curvature comparison by static and by dynamic touch. Perception \& Psychophysics 61(5) (1999) 874-894

3. Dupont, P., Hayward, V., Armstrong, B., Altpeter, F.: Single state elasto-plastic friction models. IEEE Transactions on Automatic Control 47(5) (2002) 787-792

4. Armstrong, B., Neevel, D., Kusik, T.: New results in npid control: Tracking, integral control, friction compensation and experimental results. IEEE Transactions on Control Systems Technology 9(2) (2001) 399-406

5. Hayward, V., Cruz-Hernandez, J.M.: Parameter sensitivity analysis for design and control of force transmission systems. Journal of Dynamic Systems, Measurement and Control, Transactions of the ASME 120(2) (1998) 241-248

6. Coren, S.: The left-hander syndrome: The causes and consequences of lefthandedness. Vintage Books, New York (1993)

7. Goodwin, A.W., John, K.T., Marceglia, A.H.: Tactile discrimination of curvature by humans using only cutaneous information fron the fingerpads. Experimental Brain Research 86(3) (1991) 663-672

8. Provancher, W.R., Cutkosky, M.R., Kuchenbecker, K.J., Niemeyer, G.: Contact location display for haptic perception of curvature and object motion. International Journal of Robotics Research 24(9) (2005) 691-702 